\title{
Influence of Amalgam, Alloy, and Mercury on the In Vitro Growth of Streptococcus mutans: I. Biological Test System
}

\author{
LOYS J. NUNEZ, GOTTFRIED SCHMALZ,* and JOHN HEMBREE \\ Department of Biomaterials, College of Dentistry, and Materials Science Toxicology \\ Laboratories, College of Pharmacy, University of Tennessee Center for the \\ Health Sciences, Memphis, Tennessee 38103, USA
}

$A$ procedure is presented for the in vitro growth of Streptococcus mutans in a dextrosebeef extract medium. Growth was estimated spectrophotometrically. The amount of amalgam, alloy, or mercury that was added to the sealed test tube influenced the rate and extent of growth.

The association of the plaque-forming microorganism Streptococcus mutans with dental caries in both animal and human subjects is well established ${ }^{1}$ although the exact mechanism for caries formation is not fully understood.

Perhaps the most commonly used restorative material is dental amalgam. In addition to silver and mercury, the alloys certified by the American Dental Association (ADA) that comprise amalgam contain about onepart tin to three-parts silver and small amounts of copper and zinc. 2

Differences in the incidence of secondary caries associated with restorations that contain different materials are reported ${ }^{3}$; about $9 \%$ secondary caries are reported after amalgam restoration. Noonan ${ }^{4}$ has reported that silver amalgam is not antibacterial, but other investigators have reported contrary results.5,6 For some bacteria and some metal ions, low concentrations of the metal ion may accelerate or be compatible with bacterial growth, although higher concentrations may inhibit growth.

The purpose of this investigation was to develop a procedure to allow estimation of

This research was supported, in part, by Grant No. 2434704606-R74 of the L. G. Noel Memorial Fund.

Received for publication February 13, 1975.

Accepted for publication September 10, 1975.

* Present address: ZMK Klinik, 74 Tübingen, Osianderstrasse 2-8, West Germany. the effect of amalgam and its components on $S$ mutans growth in vitro, under controlled experimental conditions.

\section{Materials and Methods}

BACTERIUM. $-S$ mutans HS-6a was used in all of the studies. This oral strain was received frozen and kept several months in the frozen state. Before being used in the experiments, portions were thawed, placed in the medium, and incubated at $37 \mathrm{C}$. After thawing, a subculture of the strain was made in a new medium every second day during the first week. The same procedure was followed daily during the second week. The bacteria were then ready to be used in the experiments.

Medium.-The medium used to make the stock culture and for the growth experiments consisted of $3 \mathrm{gm}$ of beef extract $\mathrm{t}^{\mathrm{b}}$ and 20 gm of dextrose brothc per liter of distilled deionized water. Before use, the medium was sterilized by autoclaving 15 minutes at slow exhaust.

TEST AGENTs.-A spherical alloyd in pellet form was used for amalgamation. This material is listed in the ADA List of Certified Dental Materials. Triple-distilled mercury was used in all amalgamations. ${ }^{e}$ An ultraviolet lamp, ${ }^{\mathrm{f}}$ encased in a small box $(6 \times 6 \times$ 9 inches), was used to sterilize the metal

applied by Dr. Albert T. Brown, School of Dental Medicine, Hartford, Conn.

b Difco Bacto beef extract, standardized, Difco, Detroit, Mich.

c Difco Bacto-Dextrose broth, dehydrated, Difco, Detroit, Mich.

d Batch 11431309, Kerr Manufacturing Co., Detroit, Mich.

e Rx Mercury, ACS-VSP-CP-NF, Codesco Inc., Phoenix, Ariz.

I Mineralight Ultra Violet Lamp, Ultra-Violet Products, Inc., San Gabriel, Calif. 
samples. The samples were placed about 6 inches below the surface of the lamp. The short wave length range of the lamp was used.

SAMPLE PREPARATION.-The appropriate amount of mercury was added to the alloy by means of a dispensers adjusted to give $50 \%$ mercury. For all amalgams, trituration was done mechanically ${ }^{\text {h }}$ for 18 seconds, with two pellets and the appropriate amount of mercury in the capsule. After trituration, slightly flattened cylinders $1 \mathrm{~mm}$ in diameter and about $3.5 \mathrm{~cm}$ in length were prepared for the largest samples by rolling the amalgam in a sheet of filter paper. Appropriate amounts of the amalgam were obtained by cutting different lengths of the cylinder before setting. These cylinders were then placed under the ultraviolet lamp for 20 minutes on each side.

The alloy was used as it came from the producer. Appropriate amounts were obtained by cutting the pellets. The sterilization procedure was the same as the procedure used for the amalgams.

Test Procedure.-Ten milliliters of medium was pipetted into $15 \times 125$-mm test tubes. ${ }^{i}$ Sterilization was done by autoclaving for 15 minutes at slow exhaust, and the tubes and contents were allowed to cool to $37 \mathrm{C}$. They were then inoculated with an 18- to 20-hour-old culture of $S$ mutans by pipetting $1 \mathrm{ml}$ culture into each test tube under a laminar flow hood. The test metals were also put in each test tube under asceptic conditions. The test tubes were sealed with sterile rubber stoppers, the tops covered with sterile aluminum foil, and all samples were incubated at $37 \mathrm{C}$. The experimental controls used included medium, no bacteria, no metals; medium plus bacteria, no metals; and medium plus metals, no bacteria.

Spectrophotometric readings ${ }^{j}$ expressed as percentage of transmittance $(\% T)$ at 520 $\mathrm{nm}$ were used to estimate growth. Normal saline was used as the $100 \%$ transmittance standard. Before these readings were taken, each test tube was placed on a mechanical mixer to ensure homogeneity of the liquid phase. The first reading was taken im-

g Kerr Spheraloy Proportioner, Kerr Manufacturing Co., Detroit, Mich.

h Wig-L-Bug Amalgamator, Crescent Dental Mfg. Co., Chicago, Ill.

i Corning Pyrex Laboratory Test Tubes, Cat. No. 9800 , Corning Glass Works, Corning, NY.

I Spectronic 20, Bausch and Lomb, Rochester, NY.

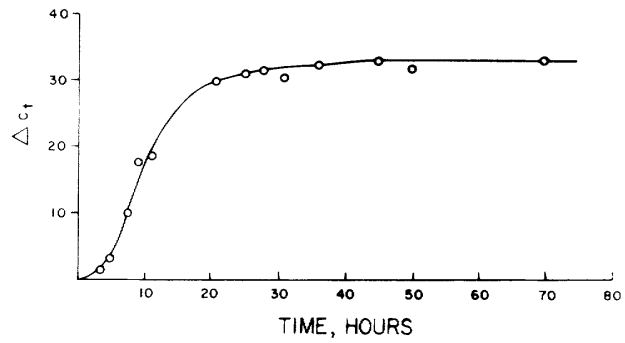

FIG 1.-Growth characteristic of $S$ mutans at 37 C.

mediately before incubation. Test tubes were inserted directly into the spectrophotometer without breaking their seals.

GROWTH ABSORBANCE RELATIONSHIP.-The relationship of absorbance to dry weight of bacteria and metabolic products was determined by filtering the bacterial suspensions through disposable filter units ${ }^{\mathrm{k}}$ that were dried in a vacuum oven at $55 \mathrm{C}$ for two hours before weighing on an analytical balance.

Data TREATMENT.-Subscripts $o, t$, and $f$ refer to initial time, time $t$, and final time, respectively. Greek letters refer to uninoculated samples. Capital letters refer to spectrophotometric readings expressed as absorbance, and lower case letters to spectrophotometric readings expressed as $\% T$. Therefore, $c_{t}=\% T$ of inoculated control (no metal) at time, $t$;

$a_{t}=\% T$ of inoculated alloy or amalgam at time, $t$;

$\lambda_{t}=\% T$ of uninoculated alloy or amalgam at time, $t$; and,

$\gamma_{t}=\% T$ of uninoculated control (medium only) at time, $t$.

Similarly $C_{t}, A_{t}, \Lambda_{t}$, and $\Gamma_{t}$ may be defined

${ }^{k}$ Millex $_{\mathrm{TM}}$ Disposable Filter Unit $(0.22 \mu \mathrm{m})$, Millipore Corp., Bedford, Mass.

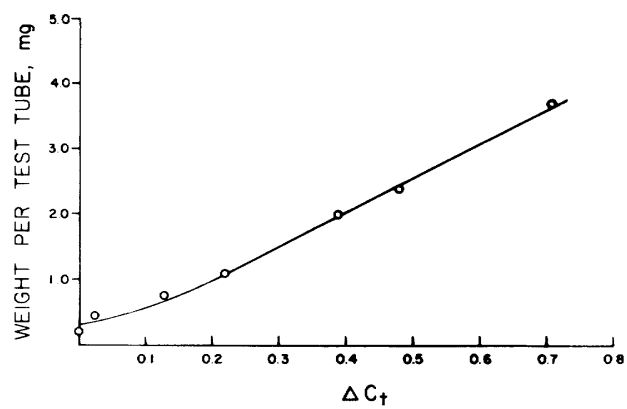

Fig 2.-Dry weight of bacteria vs absorbance. 


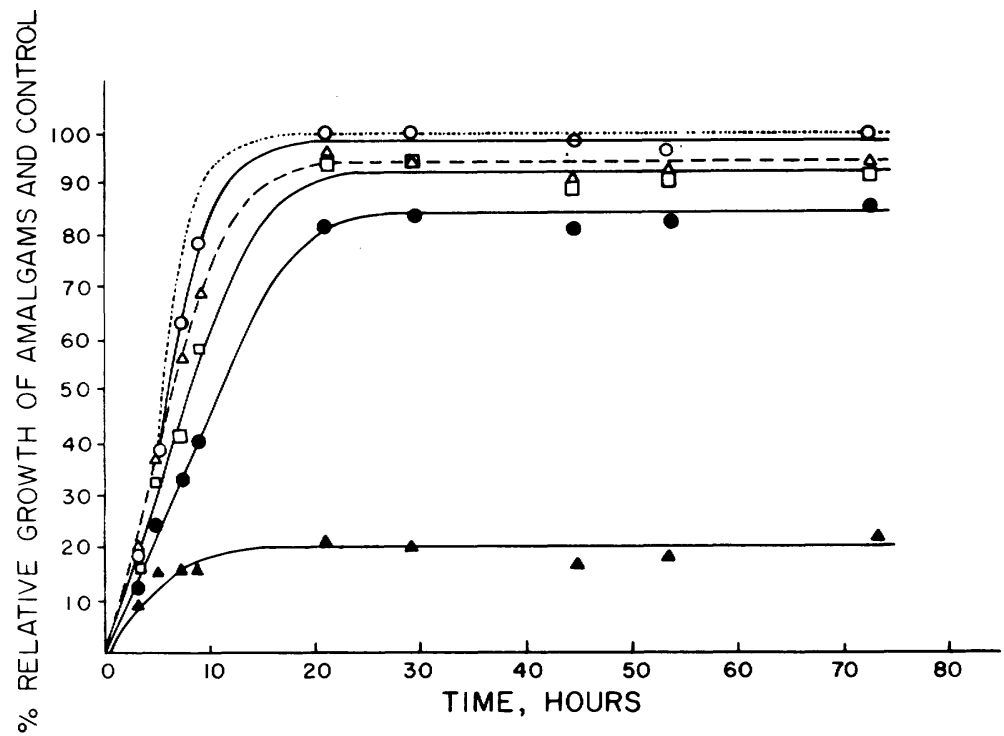

Fig 3.-Influence of amalgam on growth. Amalgam sample weight per test tube: open circles, $0.16 \mathrm{gm}$; open triangles, $0.39 \mathrm{gm}$; open squares, $0.78 \mathrm{gm}$; solid circles, $1.17 \mathrm{gm}$; and, solid triangles, $1.56 \mathrm{gm}$. Dashed line indicates inoculated control (no metal).

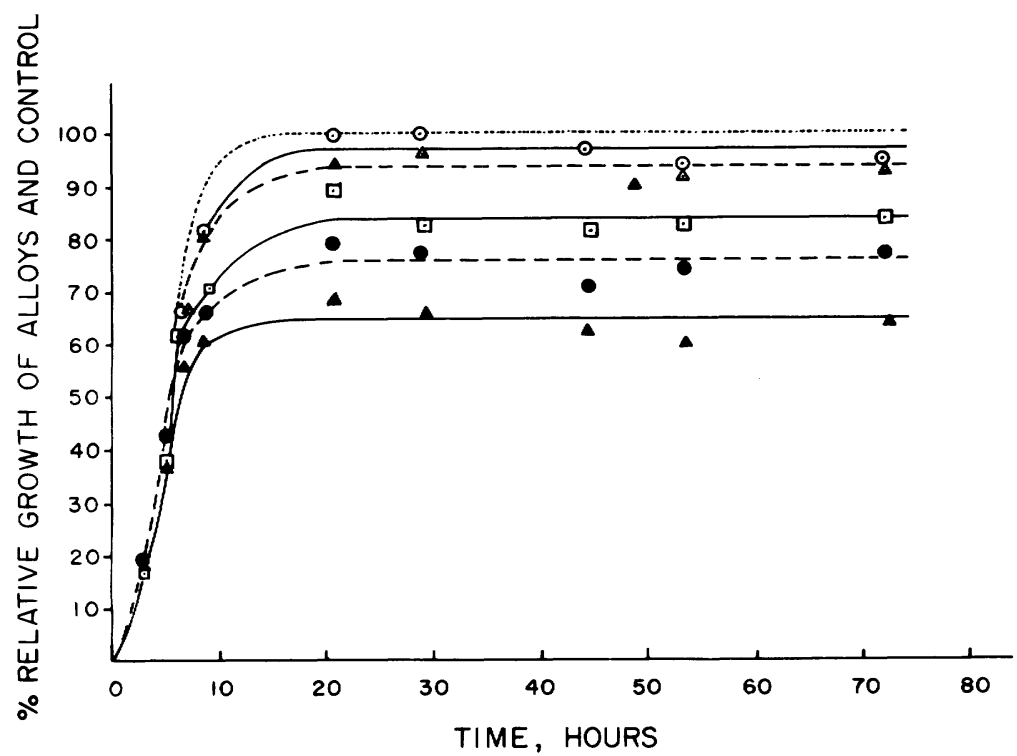

Fig 4.-Influence of alloy on growth. Alloy sample weight per test tube: open circles, $0.08 \mathrm{gm}$; open triangles, $0.19 \mathrm{gm}$; open squares, $0.39 \mathrm{gm}$; solid circles, $0.58 \mathrm{gm}$; solid triangles, $0.78 \mathrm{gm}$. Dashed line indicates inoculated control (no metal). 
in terms of absorbance rather than $\% T$. Furthermore, the equations

$$
\begin{gathered}
\Delta c_{t}=c_{o}-c_{t}-\gamma_{o}+\gamma_{t} \\
\Delta \mathrm{a}_{t}=a_{o}-a_{t}-\lambda_{o}+\lambda_{t},
\end{gathered}
$$

where $\Delta c_{t}$ and $\Delta a_{t}$ represent increased bacterial growth greater than the initial inoculation for controls (no metal) and alloys or amalgams, corrected for changes in the medium that are simultaneously occurring. Spectrophotometric readings are expressed as $\% T$. The following relationships hold when absorbance $(A)$ rather than $\% T$ is used:

$$
\begin{gathered}
\Delta C_{t}=-C_{o}+C_{t}+\Gamma_{o}-\Gamma_{t} \\
\Delta A_{t}=-A_{o}+A_{t}+\Lambda_{o}-\Lambda_{t} .
\end{gathered}
$$

The quantities $\Delta c_{\mathrm{t}}, \Delta a_{\mathrm{t}}, \Delta C_{\mathrm{t}}$, and $\Delta A_{\mathrm{t}}$ are so defined that positive values are obtained corresponding to bacterial growth for spectrophotometric readings expressed as either $\% T$ or absorbance.

The growth of the inoculated control group (no metal) may be expressed as a percentage of final growth:

and

$$
\%_{0} R c_{t}=\frac{\Delta c_{t} \times 100}{\Delta c_{f}}
$$

$$
\%^{R} C_{t}=\frac{\Delta C_{t} \times 100}{\Delta C_{f}}
$$

where $\% R c_{t}$ and $\% R C_{t}$ represent relative growth of control groups expressed as a percentage of final growth based on $\% T$ and absorbance measurements, respectively.

The growth of bacteria in the presence of amalgams or alloys may be expressed as a percent of final growth of the control groups (no metal),

and

$$
{ }_{0} R a_{t}=\frac{\Delta a_{t} \times 100}{\Delta c_{f}}
$$

$$
{ }_{0} R A_{t}=\frac{\Delta A_{t} \times 100}{\Delta C_{f}}
$$

where $\% R a_{t}$ and $\% R A_{t}$ represent bacterial growth in the presence of amalgams or alloys expressed as a percent of control growth based on $\% T$ and absorbance data, respectively.

\section{Results}

The medium proved to be suitable for growth of the HS-6 strain of $S$ mutans at 37 C. A typical, normal growth curve is shown in Figure 1. The relationship of spectrophotometric data to the total dry weight of bacteria and metabolic products is shown in Figure 2.

The data seen in Figures 3 and 4 represent growth in the presence of amalgams and alloys, respectively, expressed as the fraction of the final normal growth, based on $\% T$ data.

\section{Discussion}

Thioglycollate medium is commonly used to grow $S$ mutans in vitro. However, the thioglycollate is frequently added to inactivate mercurial preservatives. Since the effect of amalgams on bacterial growth was the object of the study, it appeared that thioglycollate medium was not suitable for our purposes.

\section{Conclusions}

Bacto-Dextrose broth with beef extract is recommended as a superior medium for cultivation of streptococci. ${ }^{7}$ This medium provided a suitable environment for growth of the HS-6 strain of $S$ mutans.

Spectrophotometric measurements that were used in our test procedure provide a simple, quick, and nondestructive means of monitoring the system during the growth period without major disturbance. The curve in Figure 2 shows that absorbance data are directly related to growth expressed as dry weight of bacteria and metabolic products.

When the data are expressed as $\% T$ vs dry weight, a curve is obtained (not shown) that is not as linear as the plot shown in Figure 2. However, when growth in the presence of the test sample is expressed as relative growth, that is, the ratio of the test sample growth to final growth of the control, there is little difference between growth curves that are based on absorbance and $\% T$ data.

It can be seen in Figures 3 and 4 that the growth response of the test procedure is sensitive to the varying amounts of amalgam or alloy added. It therefore provides an acceptable means for comparison of different amalgams on the in vitro growth of a strain of $S$ mutans.

\section{References}

1. Streptococcus mutans and Dental Caries, Proceedings of A Round Table Discussion, May 10, 1973, 73rd Annual Meeting, American Society of Microbiology Miami Beach, Florida, 
Bethesda, Md: Department of Health, Education, and Welfare Publication No. (NIH) 74-286.

2. Phillips, R.W.: Skinner's Science of Dental Materials, 7th ed, Philadelphia: W. B. Saunders Co., 1973, p 304.

3. Norman, R.D.; Mehra, R.V.; SWARTZ, M.L.; and Phillips, R.W.; Effects of Restorative Materials on Plaque Composition, J Dent Res 51: 1596-1601, 1972.

4. Noonan, R.G.: Silver Amalgam is Not AntiBacterial, J Dent Child 32: 147-153, 1965.
5. Shay, D.E.; Allen, T.J.; and Mantz, R.F.: The Anti-Bacterial Effects of Some Dental Restorative Materials, J Dent Res 35: 25-32, 1956.

6. MCCue, R.W.; McDougal, F.G.; and Shary, D.E.: The Anti-Bacterial Properties of Some Dental Restorative Materials, Oral Surg 4: 1180-1184, 1951.

7. Difco Manual of Dehydrated Culture Media and Reagents for Microbiological and Clinical Laboratory Procedures, 9th ed, Detroit: Difco Laboratories, Inc., 1974, pp 101 and 198. 